\title{
Application of Microneedle Arrays for Enhancement of Transdermal Permeation of Insulin: In Vitro Experiments, Scaling Analyses and Numerical Simulations
}

\author{
V. Leeladurga, ${ }^{1}$ U. Chandra Teja, ${ }^{1}$ S. K. Ashraf Sultana, ${ }^{1}$ K. Sudeep, ${ }^{1}$ V. Sai Sri Anusha, ${ }^{1}$ Tao Han, ${ }^{2}$ \\ Buchi N. Nalluri, ${ }^{1,3}$ and Diganta B. Das ${ }^{2,3}$
}

Received 12 August 2015; accepted 16 September 2015; published online 29 September 2015

\begin{abstract}
The aim of this investigation is to study the effect of donor concentration and microneedle (MN) length on permeation of insulin and further evaluating the data using scaling analyses and numerical simulations. Histological evaluation of skin sections was carried to evaluate the skin disruption and depth of penetration by MNs. Scaling analyses were done using dimensionless parameters like concentration of drug $\left(C_{\mathrm{t}} / C_{\mathrm{s}}\right)$, thickness $(h / L)$ and surface area of the skin $\left(S_{\mathrm{a}} / L^{2}\right)$. Simulation studies were carried out using MATLAB and COMSOL software to simulate the insulin permeation using histological sections of $\mathrm{MN}$-treated skin and experimental parameters like passive diffusion coefficient. A 1.6-fold increase in transdermal flux and 1.9-fold decrease in lag time values were observed with $1.5 \mathrm{~mm} \mathrm{MN}$ when compared with passive studies. Good correlation $\left(R^{2}>0.99\right)$ was observed between different parameters using scaling analyses. Also, the in vitro and simulated permeations profiles were found to be similar $\left(f_{2} \geq 50\right)$. Insulin permeation significantly increased with increase in donor concentration and MN length $(p<0.05)$. The developed scaling correlations and numerical simulations were found to be accurate and would help researchers to predict the permeation of insulin with new dimensions of $\mathrm{MN}$ in optimizing insulin delivery. Overall, it can be inferred that the application of MNs can significantly enhance insulin permeation and may be an efficient alternative for injectable insulin therapy in humans.
\end{abstract}

KEY WORDS: histological studies; insulin skin permeation; microneedle arrays; numerical simulations; scaling analyses.

\section{INTRODUCTION}

Diabetes mellitus is a metabolic disorder, which is characterized by insufficient production of insulin (type I) or failure of the body to utilize the insulin produced (type II). Currently, the number of patients suffering from diabetes mellitus is about 285 million adults worldwide, and by the year 2030, the numbers are expected to reach 439 million adults (1). Insulin, a pancreatic peptide hormone, is used in the treatment of insulin-dependent diabetes mellitus (IDDM or type I DM). Insulin is a water soluble, unstable protein with a molecular size of more than $5000 \mathrm{kDa}$.

Insulin cannot be administered orally as it is severely degraded in the GIT by the process of digestion (2). Drug delivery via the parenteral route, either by subcutaneous or intramuscular injection, is particularly common. Injection by hypodermic needle has been 'the gold standard' for insulin delivery for over a century (3). However, administration of insulin by hypodermic needles often causes pain, peripheral hyperinsulinemia, smooth

\footnotetext{
${ }^{1}$ Department of Pharmaceutics, KVSR Siddhartha College of Pharmaceutical Sciences, Vijayawada, 520010, AP, India.

${ }^{2}$ Department of Chemical Engineering, Loughborough University, Loughborough, Leicestershire, LE11 3TU, UK.

${ }^{3}$ To whom correspondence should be addressed. (e-mail: buchinalluri@yahoo.com; D.B.Das@lbobo.ac.uk)
}

muscle cell proliferation and a diabetic micro- and macroangiopathy and may transmit pathogens through needle re-use and require medical expertise $(4,5)$. In addition, the burden of daily injections, patient non-compliance, physiological stress, inconvenience, cost and the localized deposition of insulin leads to a local hypertrophy and fat deposition in the injection sites. To alleviate these drawbacks, transdermal route for insulin delivery can be used as an alternative non-parenteral route of administration to treat insulin-dependent diabetic patients. Given the very low diffusivity of insulin in stratum corneum owing to its high molecular weight and its hydrophilic nature, an efficient permeation enhancement technique must be employed to enhance the transdermal permeation of insulin.

Literature review revealed that a number of works have been reported on the transdermal delivery of insulin. Some of the works utilized various permeation enhancers alone and along with iontophoresis for insulin permeation enhancement $(6,7)$. Some works were also carried out using microneedles (MN) to enhance the transdermal delivery of insulin (8-12). While these works show the potential of using $\mathrm{MN}$ for enhancement of insulin delivery, and no works depicting a systematic approach to evaluate the overall dependence of insulin permeation on various parameters, for example the MN lengths (MN density in a patch) and insulin donor concentration, etc. have been explored in detail.

In addressing this issue, we have carried out a series of well-defined in vitro experiments for the evaluation of insulin 
transdermal permeation using (i) insulin solutions of two different strengths and volumes as donor vehicle and (ii) the effect and relative efficacy of different lengths of MN on insulin permeation enhancement. Moreover, the obtained data was subjected to mathematical treatment using scaling analyses to obtain correlations between dimensionless amount of insulin permeated $\left(C_{\mathrm{t}} / C_{\mathrm{s}}\right)$ and various other dimensionless variables of the study such as the surface area $\left(S_{\mathrm{a}} / L^{2}\right)$ and thickness $(h / L)$ of the skin based on the Buckingham $\pi$ theorem (13). Numerical simulations of the in vitro experiments using histological section images and passive diffusion coefficient values were also carried out in order to simulate the insulin permeation process under realistic conditions and gain insights into the phenomenon of insulin transport behaviour and insulin distribution in the MN-treated skin (14). Commercially available $\mathrm{MN}$ arrays (AdminPatch $\AA$ ) with four different needle lengths $(0.6,0.9,1.2$ and $1.5 \mathrm{~mm})$ and $\mathrm{MN}$ density were utilized for the purpose of this study. Also, a new RP-HPLCPDA method was developed for the insulin analysis.

\section{MATERIALS AND EXPERIMENTAL METHODS}

\section{Materials}

The materials used for this research work include Insugen-R (Biocon, Bangalore, India), high-performance liquid chromatography (HPLC)-grade acetonitrile, water and formic acid (E. Merck, Mumbai, India), sodium chloride, isopropyl alcohol (S.D. Fine Chem. Ltd, Mumbai, India) and haematoxylin and eosin (Sigma-Aldrich, Dorset, UK). AdminPatch ${ }^{\circledR}$ arrays (0.6, 0.9, 1.2 and $1.5 \mathrm{~mm} \mathrm{MN} \mathrm{lengths)}$ were purchased from AdminMed (Sunnyvale, CA, USA). The pig ears were obtained from a local slaughter house.

\section{Methods}

\section{Analytical Method}

The insulin analysis was performed on a Shimadzu chromatographic system equipped with HPLC Shimadzu pump LC-20AD, SIL-20A auto sampler with $200 \mu \mathrm{L}$ loop volume, programmable variable wavelength PDA detector SPDM20A VP, and Inertsil $C_{18}$ column $(250 \times 4.6 \mathrm{~mm}, 5 \mu \mathrm{m})$. The HPLC system was equipped with LC-solution software to acquire and process the data. The mobile phase consisted of $0.02 \% v / v$ formic acid and acetonitrile $(40: 60 v / v)$ and pumped at a flow rate of $1.0 \mathrm{~mL} / \mathrm{min}$. The eluents were monitored at a wavelength of $215 \mathrm{~nm}$ at ambient temperature with an injection volume of $20 \mu \mathrm{L}$. An appropriate volume of the insulin stock solution was diluted with $0.9 \% \mathrm{w} / v$ sodium chloride to get the required standard solutions at a concentration range of $5-40 \mu \mathrm{g} / \mathrm{mL}$. The proposed method was validated as per ICH guidelines (15).

\section{Preparation of $0.9 \%(w / v)$ Sodium Chloride Solution}

$0.9 \mathrm{~g}$ of sodium chloride was accurately weighed and transferred to a clean and dry $100-\mathrm{mL}$ volumetric flask containing $50 \mathrm{~mL}$ of HPLC water and dissolved. The volume was made up to mark to get $0.9 \%(w / v)$ sodium chloride solution.

\section{Determination of Insulin Stability}

The stability of insulin in saline solution was studied over a period of $48 \mathrm{~h}$ by storing $1 \mathrm{~mL}$ aliquots of $30 \mu \mathrm{g} / \mathrm{mL}$ insulin in centrifuge tubes at a temperature of $37^{\circ} \mathrm{C}$. Samples were withdrawn at different time intervals for $48 \mathrm{~h}$ and analysed for insulin content by RP-HPLC-PDA method.

\section{Skin Preparation}

The ears were collected from the local abattoirs (pigs aged about 6-7 months) and the ears were transported to the laboratory in a cooling box without previous treatment. Freezing of the skin was avoided during transport. In the laboratory, the pig ears were washed carefully with water. The hair was removed from the external part of pig ear using an electrical hair clipper. Then, the full-thickness skin was separated from the external part of the pig ear using a scalpel and excess fat under the skin was removed to a thickness of $1.2 \mathrm{~mm}$ for all the skin samples. The dermis side was wiped with isopropyl alcohol to remove residual adhering fat. The pieces of the skin obtained were individually wrapped in plastic bags and stored in a deep freezer at $-20^{\circ} \mathrm{C}$ till further use.

\section{MN Treatment of Pig Ear Skin}

Prior to the permeation experiments, the frozen skin samples were brought to room temperature and then the skin pieces were carefully washed with saline solution in a petri dish. The MN arrays differing in their needle lengths $(0.6,0.9$, 1.2 and $1.5 \mathrm{~mm}$ ) were pressed over the skin pieces at a constant thumb pressure. Photographs of the MN arrays with different needle lengths were shown in Fig. 1. The MNs were periodically checked in between usage for potential damages of the needle under a stereo microscope.

\section{Histological Examination and Calculation of Penetration Depth}

The histological sections of the skin samples without and with MN treatment were observed under the PZRM-700 microscope (Quasmo, Haryana, India). Skin samples were stained with haematoxylin and eosin $(\mathrm{H} \& \mathrm{E})$ stains for visualization of skin layers and to display a clear indentation of the MN penetration. The depth of MN penetration into the skin was also calculated with the help of ToupView 3.2 Software (AmScope, Irwin, USA).

\section{In Vitro Permeation Studies}

Franz diffusion cell apparatus (Orchid Scientifics, Nasik, India) consisting of a water circulation system, a water heater and an eight-stage magnetic stirrer was used to carry out permeation studies. The diffusion cells $\left(1.77 \mathrm{~cm}^{2}\right.$ diffusional area and a receptor volume of $14 \mathrm{~mL}$ ) were employed for conducting the permeability studies and the donor compartment was charged with insulin solutions (i.e. 40 and $100 \mathrm{IU} / \mathrm{mL}$ at 0.5 and $1 \mathrm{~mL}$ donor volumes) while the receiving compartment was loaded with saline solution. 

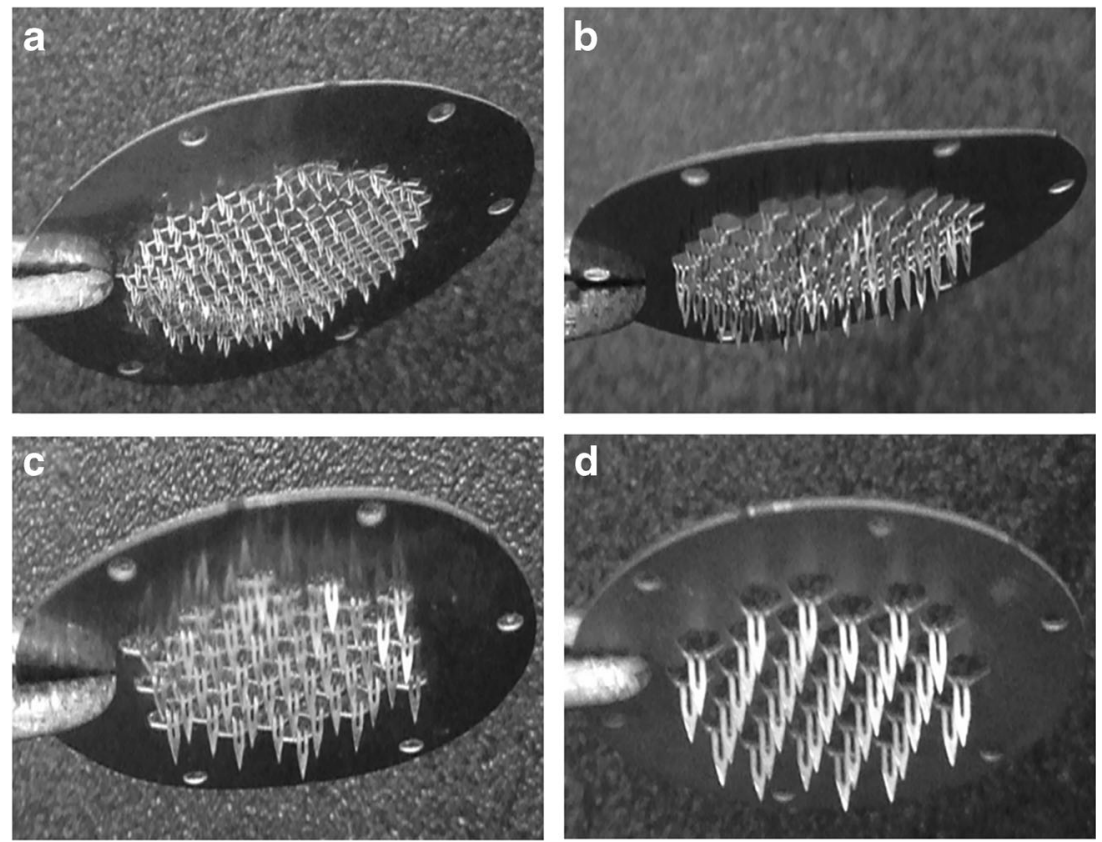

Fig. 1. Photographs of $\mathrm{MN}$ arrays—a $0.6 \mathrm{~mm}$, b $0.9 \mathrm{~mm}$, c $1.2 \mathrm{~mm}$, d $1.5 \mathrm{~mm}$

Pig ear skin was mounted between donor and receptor compartments, with the stratum corneum side facing towards the donor compartment and clamped. The receptor medium was magnetically stirred at a speed of $600 \mathrm{rpm}$ for uniform distribution of insulin. Care was taken to prevent possible entrapment of air bubbles under the skin (dermis) and in receptor solution. The surface of the skin was maintained at $32^{\circ} \mathrm{C}$ using a circulating water bath. After equilibration, insulin solution was charged on to the skin. At predetermined time points, $0.5 \mathrm{~mL}$ samples were withdrawn from the receptor fluid and replaced with fresh medium immediately. The amount of insulin in samples was analysed by RP-HPLCPDA method. The insulin skin permeation profiles were plotted for passive and MN-treated (0.6, 0.9, 1.2 and $1.5 \mathrm{~mm})$ skin permeation studies. The flux values and respective lag time values were obtained from the slope and X-intercept of the steady-state portion of the permeation profiles.

\section{Insulin Content in the Skin}

Insulin retained within the skin was measured at the end of the experiment. The skin tissue exposed to the donor solution was cut with a scalpel and rinsed with water and blotted with a paper towel in order to remove the adhered insulin to the surface. The skin was then minced with a scalpel and placed in a pre-weighed vial. The insulin was extracted from the skin by equilibrating with $1 \mathrm{~mL}$ of acetonitrile at room temperature on an orbital shaker (60 rpm). Samples were analysed by HPLC to determine the insulin content.

\section{Scaling Analyses}

Scaling analyses were performed using the amount of insulin permeated $\left(C_{\mathrm{t}} / C_{\mathrm{s}}\right)$ and other variables of the study like the surface area $\left(S_{\mathrm{a}} / L^{2}\right)$ and thickness $(h / L)$ of the skin using the Buckingham $\pi$ theorem where it is defined that the dimensionless concentration of a drug, which permeates through skin sample, can be expressed in terms of key non-dimensional parameters, e.g. MN lengths (13). Full details of the approach for the nondimension analyses have been presented earlier (13), and therefore, they are not discussed in detail in this paper. Equation 1 describes the relationship of all the parameters used for such analyses and the various method parameters used in this study for scaling analyses are given in Table I.

$\frac{C_{\mathrm{t}}}{C_{\mathrm{s}}}=K\left[\frac{S_{\mathrm{a}} L^{4} K_{\mathrm{e}}}{V_{\mathrm{d}} h D}\right]^{n}$

Where $K$ is a dimensionless constant and $n$ is an unknown power, $C_{\mathrm{t}}$ and $C_{\mathrm{s}}$ are the concentration of the drug permeated at a given time $t(6 \mathrm{~h})$ and the concentration of the drug loaded for diffusion (surface concentration on the skin), $S_{\mathrm{a}}$ is the surface area of the patch or MN-treated area of the skin, $L$ is the length of MN, $K_{\mathrm{e}}$ is the first order elimination constant of the drug, $V_{\mathrm{d}}$ is the volume of receptor fluids (in vitro), $h$ is the thickness of the skin and $D$ is the diffusion coefficient of insulin in the skin. Using Eq. 1, the correlations between the groups referring to the dimensionless insulin concentration $\left(C_{\mathrm{t}} / C_{\mathrm{s}}\right)$ against different dimensionless parameters of the study, $h / L$ and $S_{\mathrm{a}} / L^{2}$, have been established considering that all other variables remain unchanged.

\section{Numerical Simulation of Permeation Experiments}

Simulations were carried out using a MATLAB (Math Works, MA, USA) program, which was employed to prepare the microphotographs of histological sections of the skin treated with MN. These images, coupled with the experimental parameters, e.g. passive diffusion coefficient obtained from the in vitro studies were imported into a simulator software, COMSOL Multiphysics (COMSOL Multiphysics Pvt. Ltd., 
Table I. Model Parameters for Dimensional Analyses of Data

\begin{tabular}{ll}
\hline Parameters & Value \\
\hline Duration for medication (calculation): $t_{\mathrm{m}}(\mathrm{h})$ & 6 \\
Surface area of skin exposed for permeation: $S_{\mathrm{a}}\left(\mathrm{cm}^{2}\right)$ & 1.77 \\
Thickness of stratum corneum: $h_{\mathrm{sc}}(\mathrm{cm})$ & 0.002 \\
Total thickness of membrane (distance to blood vessel): & 0.12 \\
$\quad h(\mathrm{~cm})$ & \\
Effective skin thickness: $h_{\mathrm{e}}(\mathrm{cm})$ & Variable \\
Diffusion coefficient in viable skin: $D_{\mathrm{vs}}\left(\mathrm{cm}^{2} / \mathrm{s}\right)$ & Variable \\
Volume of receptor compartment $(\mathrm{distribution}): V_{\mathrm{d}}(\mathrm{mL})$ & 14 \\
Skin surface/donor concentration: $C_{\mathrm{s}}(\mathrm{mg} / \mathrm{mL})$ & 3.5 \\
MN length: $L(\mathrm{~cm})$ & Variable \\
\hline$M N$ microneedle &
\end{tabular}

Stockholm, Sweden), in order to simulate the insulin transport behaviour across the skin and predict the permeation profiles of insulin as per the procedures reported by Han and Das (14).

\section{Statistical Analysis of the Data}

Statistical analysis of the data and different parameters obtained with skin permeation experiments were computed with a one-way ANOVA analysis (Fischer's LSD post hoc test) using SYSTAT 13 software (Systat Software Inc., CA, USA). Results with $p$ value less than 0.05 were considered to be a statistically significant variance.

\section{RESULTS}

\section{Analytical Method}

In the present investigation, a rapid, efficient RP-HPLCPDA method was developed for the quantitative estimation of insulin. The method was validated according to the $\mathrm{ICH}$ guidelines and it complies with all specifications (15). The validation data was given in Table II. Insulin eluted at $6.08 \mathrm{~min}$, which showed a good linearity in the concentration range of $5-40 \mu \mathrm{g} / \mathrm{mL}$ with a correlation coefficient of 0.999 . The percent recoveries ranged between 98 and $102(\mathrm{RSD}<2)$.

Table II. Linearity, Precision and Accuracy Data of Insulin Linearity $(n=3)$

\begin{tabular}{ll}
\hline Range & $5-40 \mu \mathrm{g} / \mathrm{mL}$ \\
Regression equation & $y=2307 x-20,464$ \\
Correlation coefficient & $R=0.998$ \\
Regression coefficient & $R^{2}=0.996$ \\
Precision $(n=6)$ & Average peak area of the \\
& standard sample (\%RSD) \\
& $663,000(1.9)$ \\
Accuracy $(n=3)$ & Mean percent recovery \\
$\%$ level of addition & $(\%$ RSD) \\
80 & $101.3(0.351)$ \\
100 & $100.9(0.433)$ \\
120 & $101.6(1.028)$ \\
\hline
\end{tabular}

$R S D$ relative standard deviation

\section{Determination of Insulin Stability}

Stability of insulin in $0.9 \% w / v$ saline solution, over a period of $48 \mathrm{~h}$ was studied and the data revealed that the percent variation in insulin content at different time intervals was found to be less than $2 \%$. The results of the stability studies showed good insulin stability over the entire experimental duration with no significant degradation.

\section{Histological Examination and Calculation of Penetration Depth}

Histological sections were prepared using haematoxylin and eosin stain and representative microscopic images were shown in Fig. 4a. The photographs clearly indicated the stratum corneum barrier disruption and the formation of microconduits across the skin layers. The representative penetration depth values for the MNs used were calculated using ToupView ${ }^{\circledR}$ software and were found to be approximately 363.25, 521.16 and $642.34 \mu \mathrm{m}$ for $0.6,1.2$ and $1.5 \mathrm{~mm} \mathrm{MN}$ lengths.

\section{In Vitro Permeation Studies}

Passive in vitro permeation studies were carried out using 40 and $100 \mathrm{IU} / \mathrm{mL}$ strength insulin solutions at 0.5 and $1.0 \mathrm{~mL}$ donor volumes. The amount of insulin permeated through the skin at different time intervals was determined by the RPHPLC-PDA method. The cumulative amounts of insulin permeated through intact pig ear skin at the end of $6 \mathrm{~h}$ were found to be $329.66 \pm 23.78$ and $355.93 \pm 21.17 \mu \mathrm{g} / \mathrm{cm}^{2}$, respectively, for $0.5 \mathrm{~mL}$ and $444.19 \pm 19.53$ and $452.84 \pm 27.57 \mu \mathrm{g} / \mathrm{cm}^{2}$, respectively, for $1 \mathrm{~mL}$ of 40 and $100 \mathrm{IU} / \mathrm{mL}$ insulin solutions. On the other hand, the steady-state flux values were found to be $71.66 \pm 3.72$ and $76.95 \pm 3.86 \mu \mathrm{g} / \mathrm{cm}^{2} / \mathrm{h}$ for $0.5 \mathrm{~mL}$ donor volume and $91.05 \pm 6.66$ and $92.12 \pm 6.75 \mu \mathrm{g} / \mathrm{cm}^{2} / \mathrm{h}$ for $1 \mathrm{~mL}$ donor volume, respectively, for 40 and $100 \mathrm{IU} / \mathrm{mL}$ strength insulin solutions. Hence, for further MN-treated studies, $1 \mathrm{~mL}$ of $100 \mathrm{IU} / \mathrm{mL}$ strength insulin solution was used as donor vehicle. The cumulative amount of insulin permeated after treatment with $0.6,0.9,1.2$ and $1.5 \mathrm{~mm} \mathrm{MN}$ were found to be $536.35 \pm 37.66,624.27 \pm 39.16,734.28 \pm 45.27$ and 789.20 $\pm 38.33 \mu \mathrm{g} / \mathrm{cm}^{2}$, respectively, and the respective flux values were $105.59 \pm 6.23,123.6 \pm 2.22,140.67 \pm 4.67$ and 146.44 $\pm 3.83 \mu \mathrm{g} / \mathrm{cm}^{2} / \mathrm{h}$. The permeation parameters viz lag times, permeability coefficients, diffusion coefficients and insulin content in the skin were given in Table III and the comparative permeation profiles are shown in Fig. 2.

\section{Scaling Analyses}

It is well known that many parameters of MN, skin and the drug have great influence on the overall permeation enhancement achieved by this technique (8). Using scaling analyses, the effect of MN length with respect to dimensionless skin thickness $(h / L)$ and dimensionless surface area of the skin $\left(S_{\mathrm{a}} / L^{2}\right)$ on the dimensionless amount of insulin permeated $\left(C_{\mathrm{t}}\right)$ $C_{\mathrm{s}}$ ) were determined (Fig. 3a, b, respectively). Equations 2 and 3 describe such relationships between the considered dimensionless groups within the given range. 
Table III. In Vitro Transdermal Permeation Parameters of Insulin

\begin{tabular}{|c|c|c|c|c|c|}
\hline \multirow[t]{2}{*}{ Parameter } & \multicolumn{5}{|l|}{ Treatment } \\
\hline & Passive & $0.6 \mathrm{~mm} \mathrm{MN}$ & $0.9 \mathrm{~mm} \mathrm{MN}$ & $1.2 \mathrm{~mm} \mathrm{MN}$ & $1.5 \mathrm{~mm} \mathrm{MN}$ \\
\hline Lag time $(\mathrm{h})$ & $1.13 \pm 0.11$ & $0.98 \pm 0.08$ & $0.92 \pm 0.1$ & $0.8 \pm 0.16$ & $0.59 \pm 0.13$ \\
\hline Permeability coefficient $(\mathrm{cm} / \mathrm{h}) \times 10^{-3}$ & $26.07 \pm 1.62$ & $30.16 \pm 1.77$ & $35.31 \pm 0.63$ & $40.19 \pm 1.33$ & $41.83 \pm 1.09$ \\
\hline Diffusion coefficient $\left(\mathrm{cm}^{2} / \mathrm{s}\right) \times 10^{-7}$ & $8.938 \pm 0.55$ & $10.340 \pm 0.60$ & $12.106 \pm 0.216$ & $13.779 \pm 0.456$ & $14.341 \pm 0.37$ \\
\hline Insulin content in the skin (ng/g) & $651.75 \pm 120.94$ & $843.35 \pm 139.37$ & $1688.61 \pm 291.01$ & $743.2 \pm 140.55$ & $565.49 \pm 60.69$ \\
\hline
\end{tabular}

$M N$ microneedle

$\frac{C_{\mathrm{t}}}{C_{\mathrm{s}}}=0.364\left[\frac{h}{L}\right]^{-0.434}$ for $0.8 \leq \frac{h}{L} \leq 2.0$

$\frac{C_{\mathrm{t}}}{C_{\mathrm{s}}}=1.036\left[\frac{S_{\mathrm{a}}}{L^{2}}\right]^{-0.217}$ for $78.5 \leq \frac{S_{\mathrm{a}}}{L^{2}} \leq 491.5$

These correlations were determined for given thickness and surface area of the skin while the MN length varies (0.6, $0.9,1.2$ and $1.5 \mathrm{~mm}$ ). The $R^{2}$ values for the dimensionless groups $\left(C_{\mathrm{t}} / C_{\mathrm{s}} v s h / L\right.$ and $\left.C_{\mathrm{t}} / C_{\mathrm{s}} v s S_{\mathrm{a}} / L^{2}\right)$ were found to be $\geq 0.99$.

\section{Numerical Simulations of Permeation Experiments}

Microphotographs of histological sections can provide an efficient way of predicting the extent of skin disruption by different $\mathrm{MN}$, which may be used to develop accurate simulations of permeation process. The various stages in the treatment of histological images by MATLAB and COMSOL were shown in Fig. 4.

In order to confirm the validity of the developed simulations, the permeation profiles obtained from in vitro transdermal permeation experiments and that from simulation (for $0.6 \mathrm{~mm}$ MN treatment) were compared (Fig. 5). The similarity factor $\left(f_{2}\right)$ for both the profiles was computed and was found to be 50.4.

\section{DISCUSSION}

A rapid, specific and precise RP-HPLC-PDA method was developed for the quantitative estimation of insulin and the method was validated according to $\mathrm{ICH}$ guidelines. The validation parameters like specificity, linearity, accuracy, limit

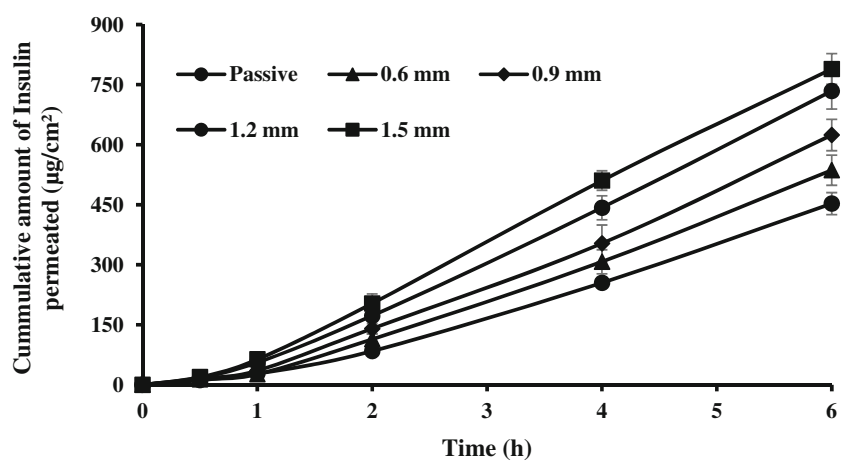

Fig. 2. Mean comparative in vitro skin permeation profiles of insulin of detection, limit of quantification, precision, robustness, system suitability and stability were all within the official compendial limits. The developed method was employed for the estimation of insulin in various samples throughout the experiment. Stability of insulin in saline solution was determined over a period of $48 \mathrm{~h}$ at $37 \mathrm{C}$ and showed no significant degradation of insulin till the end of experimental duration.

AdminPatch ${ }^{\circledR}$ arrays different lengths $(0.6,0.9,1.2$, $1.5 \mathrm{~mm}$ ) were used to study their effect on enhancement of
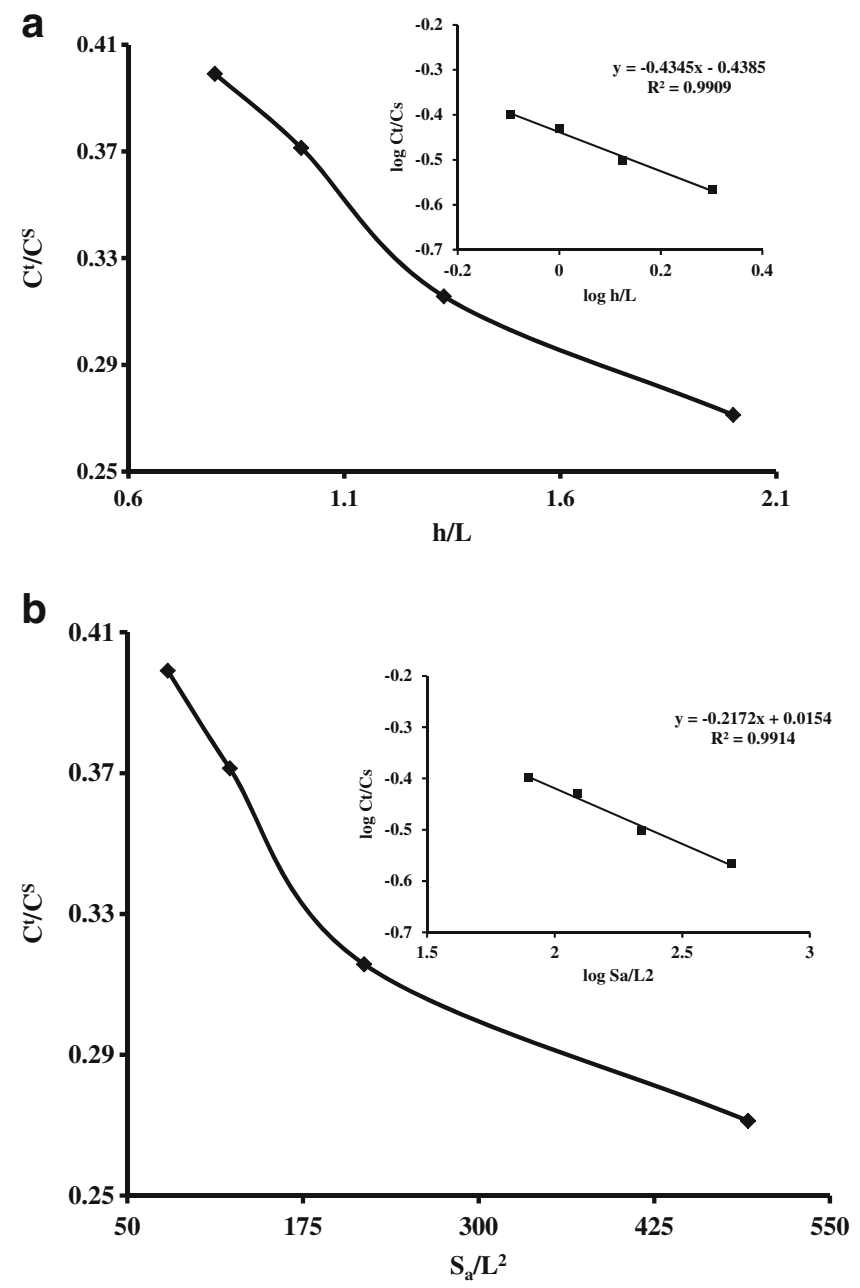

Fig. 3. Scaling relationships for permeation of insulin. a General correlation between dimensionless insulin concentration $\left(C_{\mathrm{t}} / C_{\mathrm{s}}\right)$ and dimensionless skin thickness $(h / L)$. b General correlation between dimensionless insulin concentration $\left(C_{\mathrm{t}} / C_{\mathrm{s}}\right)$ and dimensionless surface area $\left(S_{\mathrm{a}} / L^{2}\right)$ 


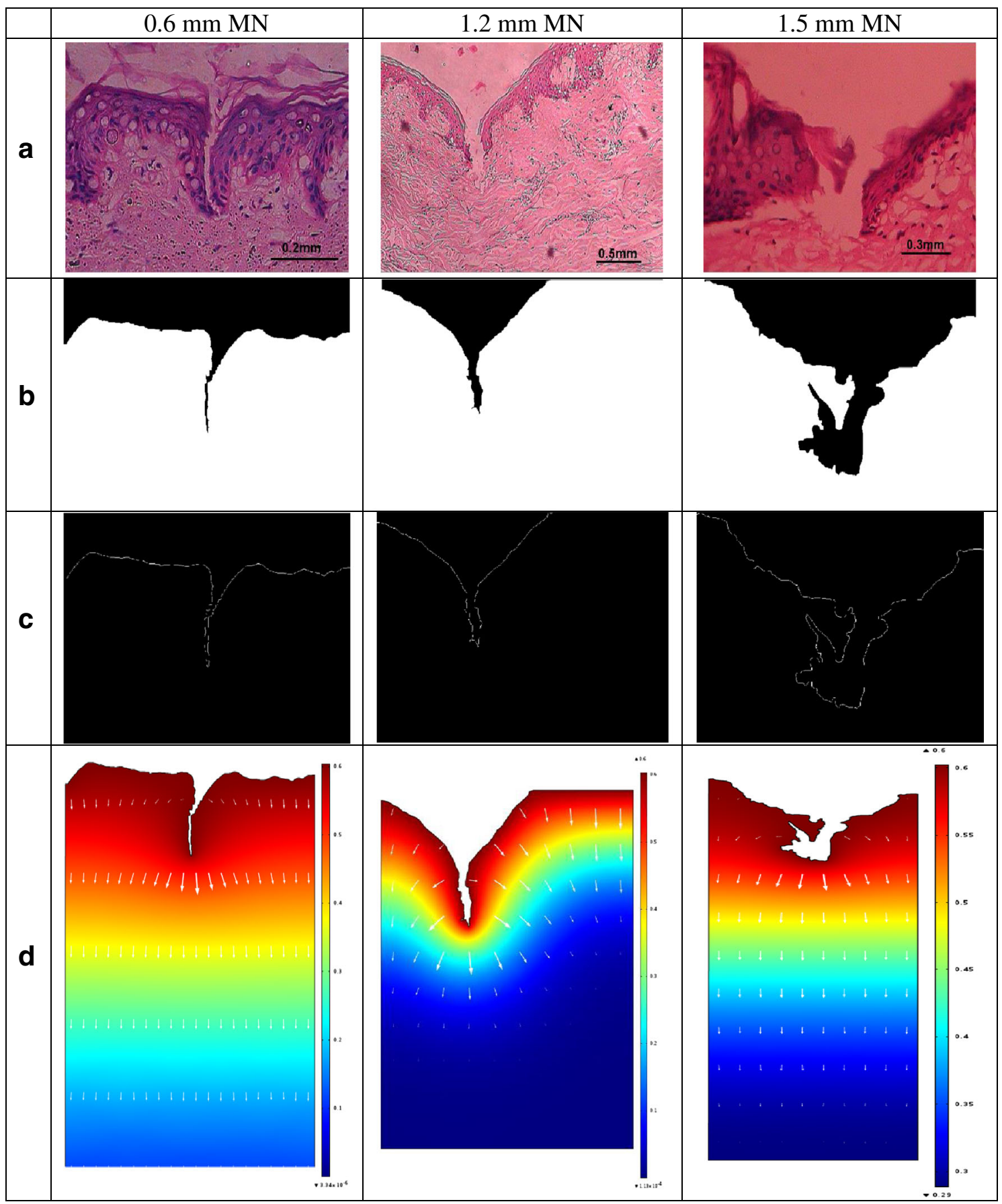

Fig. 4. a The histological section images of skin. b, $\mathbf{c}$ Images treated and processed with MATLAB program. d Images simulated in COMSOL

insulin skin permeation (Fig. 1). In order to gain insights into the microconduits formed by the MN application, histological sections (Fig. 4a) were prepared and evaluated. The MN insertions and stratum corneum disruption were clearly evident from the histological sections. The MN arrays penetrated through the cells without merely indenting them. Overall, the depth of penetration as measured by ToupView ${ }^{\circledR}$ software was found to be approximately $40-50 \%$ of the needle length and increase in needle length increased the penetration depth as supported by the microconduits formed by different lengths of MNs, i.e. 1.2 and $1.5 \mathrm{~mm}$ as shown in Fig. 4a and which may be due to the resistance shown by the skin for $\mathrm{MN}$ insertion as a result of its viscoelastic nature $(8,16)$.

In concurrence with the reports published earlier that a large amount of insulin penetrates through the skin after the application of high concentrations of insulin at the donor site, the passive diffusion experiments we conducted resulted in a 1.5 -fold increase in the cumulative amount of insulin permeated by increasing insulin strength from 40 to 100 IU and volume from 0.5 to $1 \mathrm{~mL}$ as donor vehicle $(p<0.05)(11,12)$. This increase in the insulin permeation may be attributed to increased chemical potential of insulin in donor compartment. 


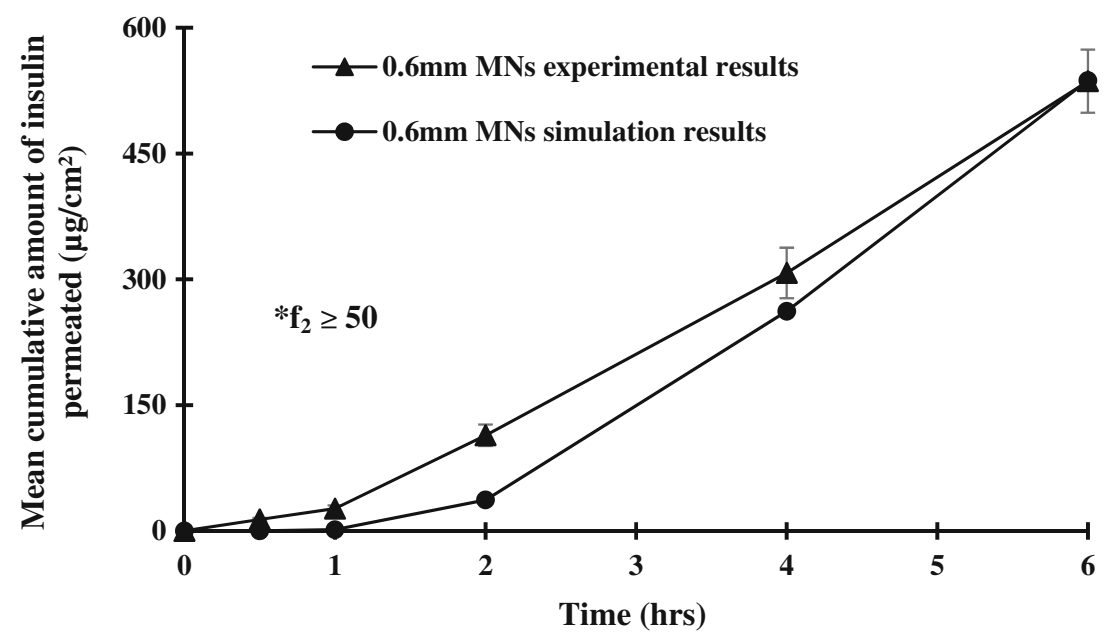

Fig. 5. Comparative transdermal permeation profiles of insulin from experimental and simulation studies

Based on the above results, $100 \mathrm{IU} / \mathrm{mL}$ strength insulin solution at $1 \mathrm{~mL}$ donor volume was used for further MN application studies, the data from which was used for comparing the relative efficacy of $\mathrm{MN}$ of different lengths on enhancement of insulin permeation and for developing non-dimensional scaling analysis and numerical simulations of the experiments so as to attain better understanding on the insulin transport behaviour after MN application.

The permeation parameters of insulin with passive and $\mathrm{MN}$ treated studies were compared (Table III). The results inferred in a 1.18-, 1.38-, 1.62- and 1.74-fold increase in the cumulative amount of insulin permeated at the end of $6 \mathrm{~h}$ for $0.6,0.9,1.2$ and $1.5 \mathrm{~mm} \mathrm{MN}$ lengths, respectively, when compared to passive permeation results. Similarly, a 1.15-, 1.34-, 1.53-, 1.6-fold increase in flux values and 1.15-, 1.23-, 1.41-, 1.9-fold decrease in lag times were observed. One-square-centimetre circular $\mathrm{MN}$ arrays $(0.6,0.9,1.2$ and $1.5 \mathrm{~mm}$ ) possess $187,85,41$ and 31 needles with effective lengths of $0.5,0.8,1.1$ and $1.4 \mathrm{~mm}$, respectively. The viable epidermis, which is a cellular, avascular tissue measuring 50-100 $\mu \mathrm{m}$ thickness, poses a significant barrier activity for transdermal delivery of drugs along with the stratum corneum and that the removal of full epidermis by calibrated microderm abrasion increased skin permeability of insulin by 11-fold (17). The ability of longer MNs (1.2 and $1.5 \mathrm{~mm})$ to create microconduits across the depth of the epidermis without microderm abrasion might explain the increased permeability of insulin even with relatively less number of pores created in the skin.

The concerns of pain and damage to small capillaries in the dermis, after application of longer MNs (1.5 mm length), can be overruled by the previously published data, which states that MN application (480 to $1450 \mu \mathrm{m}$ ) was significantly less painful to human volunteers ( 5 to $40 \%$ pain scores) when compared to 26-gauge hypodermic needle (18). Moreover, it was reported in a previous study that the skin reverted to its normal physiological structure within $8 \mathrm{~h}$ specifically after application of $1.0 \mathrm{~mm} \mathrm{MN}$ rollers, while the injury by hypodermic needle still persisted after $24 \mathrm{~h}$ in vivo using a rat model (10). The depth of MN penetrated in to the skin layers we observed was found to be $40-50 \%$ of the MN length, further strengthens the above statements that nerve damage and pain on $\mathrm{MN}$ application are less probable.

In a previous study, MNs of $800 \mu \mathrm{m}$ in length loaded with $0.13,0.25$ and $0.44 \mathrm{IU}$ of bovine insulin were fabricated from hyaluronic acid resulted in the release of insulin (>90\%) within $1 \mathrm{~h}$ in both in vitro and in vivo (rat model) permeation studies and that the delivery rate from insulin-loaded MNs was found to be comparable to that obtained with subcutaneous injection (9). Poke and solution application technique employed in our investigation using $\mathrm{MN}$ arrays of 1.2 and $1.5 \mathrm{~mm}$ length resulted in 37 and $40 \%$ permeation of insulinloaded dose at the end of $6 \mathrm{~h}$. Moreover, no saturation or plateau signs in the permeation profile were observed after $\mathrm{MN}$ application (Fig. 2). This can be extrapolated to a continuous and controlled insulin delivery via the skin under in vivo conditions for more than $6 \mathrm{~h}$.

In corroboration with the data we obtained in this investigation, an in vivo study on a rat model with a similar 'poke and solution application' technique, using $\mathrm{MN}$ rollers $(0.25$, 0.5 and $1 \mathrm{~mm}$ ), was reported by Liu et al., showing a positive relationship between the MN length and hypoglycemic effect produced thereof (10). However, a superior hypoglycemic effect in vivo may be anticipated with $\mathrm{MN}$ arrays application, as the number of MNs piercing the skin per unit area is more on arrays than on rollers.

Insulin retained within the skin was measured at the end of the experiment in order to have an idea of insulin retained in skin layers after the permeation studies (Table III). Even though there is no correlation of insulin skin content with different experiments, significant amounts of insulin was distributed in skin layers at the end of the experiments indicating potential skin deposition of insulin.

The scaling analyses were developed between various dimensionless parameters (Fig. 3a, b) in order to generalize the effect of MN length $(L)$, skin thickness $(h)$ and surface area of the skin $\left(S_{\mathrm{a}}\right)$ exposed on the overall amount of insulin permeated $\left(C_{\mathrm{t}} / C_{\mathrm{s}}\right)(13)$. A good correlation $\left(R^{2} \geq 0.99\right)$ was observed between the dimensionless parameters and can be used to predict the amount of insulin permeated $\left(C_{\mathrm{t}} / C_{\mathrm{s}}\right)$ with high accuracy for other $\mathrm{MN}$ lengths in the range of $0.6-1.5 \mathrm{~mm}$.

Besides the scaling analyses, a set of well-defined numerical simulations of the permeation experiments were carried out in order to gain better insights into the effects of $\mathrm{MN}$ design, force of insertion of $\mathrm{MN}$, thickness of the skin, etc. in enhancing the insulin permeation and also to obtain the information about insulin distribution within the skin during 
permeation, which are difficult to obtain directly from the experimental data. In order to carry out simulation studies of high accuracy, the relative ability of the MNs in creating microconduits in the skin should be understood precisely. It is well known now that owing to the viscoelastic nature of the skin, the microconduits created do not have the same dimensions as the MN, but may vary significantly $(8,19)$, thereby limiting the applicability of MN design parameters to carry out such simulations. Microphotographs of histological sections can provide an efficient way of predicting the extent of skin disruption by different MNs, which may be used to develop accurate simulations of the drug(s) permeation process (14). The numerical simulations carried out using MATLAB (Fig. 4b, c) and COMSOL (Fig. 4d) softwares, based on in vitro passive diffusion coefficient values and histological section images (of corresponding MN geometry) were able to provide information regarding drug distribution within the skin at any time point and depth (14).

The similarity factor $\left(f_{2}\right)$ for the in vitro permeation profile and the simulated profile was found to be 50.4, which indicates that the developed simulations for insulin permeation under given conditions were in good agreement with the experimental results and therefore can be used to accurately predict permeation profiles of insulin using histological skin sections treated specific shape and size of MNs.

Furthermore, these simulation studies can also be used to predict the permeation profiles of other drugs with different dimensions and designs of MNs using the respective histological section images and the passive diffusion coefficient of the drug in the skin. These scaling and simulation studies would help researchers in optimizing the MN geometry and other experimental conditions in further improving the degree of enhancement of insulin or other drugs.

\section{CONCLUSION}

Transdermal delivery of therapeutic agents like insulin can be of great clinical advantage, as it is relatively less invasive and provides a continuous delivery with low frequency of administration and thus increased patient compliance. Our study inferred in a significant enhancement in insulin permeation across the pig ear skin and is donor concentration and volume dependent. The permeation of insulin after MN application increased with an increase in needle length. The developed scaling correlations and numerical simulations can be of great research value for understanding and optimizing insulin delivery through transdermal route. Thus, developing appropriate $\mathrm{MN}$-assisted transdermal insulin delivery systems may be a promising alternative for injectable insulin therapy. However, further in vivo studies are needed to be carried out to evaluate the therapeutic efficacy of this technique and its applicability to human use.

\section{ACKNOWLEDGMENTS}

The authors are thankful to the Siddhartha Academy of General and Technical Education, Vijayawada, for providing facilities and support to carry out this research work and NRI Academy of Medical Sciences, Guntur, for providing the facilities required for the histological examination of the skin samples.

\section{Compliance with Ethical Standards}

Conflict of Interest The authors declare that they have no competing interests.

\section{REFERENCES}

1. Depreter F, Amigi K. Formulation and in vitro evaluation of highly dispersive insulin dry powder formulations for lung administration. Eur J Pharm Biopharm. 2010;76:454-63.

2. Ito Y, Hagiwara E, Saeki A, Sugioka N, Takada K. Feasibility of microneedles for percutaneous absorption of insulin. Eur J Pharm Sci. 2006;29:82-8.

3. McAllister DV, Wang PM, Davis SP, Park JH, Canatella PJ, Allen $\mathrm{MG}$, et al. Micro fabricated needles for transdermal delivery of macromolecules and nanoparticles: fabrication methods and transport studies. Proc Natl Acad Sci. 2003;100:13755-60.

4. Khafagy ES, Morishita M, Onuki Y, Takayama K. Current challenges in non-invasive insulin delivery systems: a comparative review. Adv Drug Deliv Rev. 2007;59:1521-46.

5. Gill HS, Prausnitz MR. Coated microneedles for transdermal delivery. J Control Release. 2007;117:227-37.

6. Yerramsetty KM, Neely BJ, Madihally SV, Gasem KAM. A skin permeability model of insulin in the presence of chemical penetration enhancer. Int J Pharm. 2010;388:13-23.

7. Pillai O, Vinod N, Ramesh P. Transdermal iontophoresis of insulin: IV. Influence of chemical enhancers. Int J Pharm. 2004;269:109-20.

8. Chueng K, Han T, Das DB. Effect of force of microneedle insertion on the permeability of insulin in skin. J. Diabetes Sci. Technol. 2014; 1-9.

9. Liu S, Jin MN, Quan YS, Kamiyana F, Katsumi H, Sakane T, et al. The development and characteristic of novel microneedle arrays fabricated from hyaluronic acid, and their application in the transdermal delivery of insulin. J Control Release. 2012;161(3):933-41.

10. Zhou CP, Yu-Ling L, Wang HL, Zhang PX, Zhanq JL. Transdermal delivery of insulin using microneedle rollers in vivo. Int $\mathrm{J}$ Pharm. 2010;392(1-2):127-33.

11. Martanto W, Davis SP, Holiday NR, Wang J, Gill HS, Prausnitz MR. Transdermal delivery of insulin using microneedles in vivo. Pharm Res. 2004;21:947-52.

12. Wu Y, Gao Y, Qin G, Zhang S, Qiu Y, Li F, et al. Sustained release of insulin through skin by intradermal microdelivery system. Biomed Microdevices. 2010;12:665-71.

13. Al-Qallaf B, Das DB, Mori D, Cui Z. Modelling transdermal delivery of high molecular weight drugs from microneedle systems. Phil Trans R Soc A. 2007;365:2951-67.

14. Han T, Das DB. A new paradigm for numerical simulation of microneedle-based drug delivery aided by histology of microneedle-pierced skin. J Pharm Sci. 2015;104(6):1993-2007.

15. International conference on harmonization of technical requirements for registration of pharmaceuticals for human use. Validation of text and methodology Q2 (R1), 2005.

16. Shaun DG, Chen B, Huadong X, Aleksandr O, Boris NC, Nancy $\mathrm{AM}$, et al. The effects of geometry on skin penetration and failure of polymer microneedles. J Adhes Sci Technol. 2013;27(3):227-43.

17. Samantha NA, Eunhye J, Prausnitz MR. Transdermal delivery of molecules is limited by full epidermis, not just stratum corneum. Pharm Res. 2013;30(4):1099-109.

18. Gill HS, Denson DD, Burris BA, Prausnitz MR. Effect of microneedle design on pain in human volunteers. Clin J Pain. 2008;24:585-94.

19. Gomaa YA, Morrow DJ, Garland MJ, Donnelly RF, ElKhordagui LK, Meidan VM. Effects of microneedle length, density, insertion time and multiple applications on human skin barrier function: assessments by trans-epidermal water loss. Toxicol In Vitro. 2010;24:1971-8. 